\title{
Multicast Tree Construction with QoS Guaranties
}

\author{
O. Moussaoui ${ }^{1}$, A. Ksentini $^{1}$, M. Naïmi ${ }^{1}$, and A. Gueroui ${ }^{2}$ \\ ${ }^{1}$ LICP EA 2175, Université de Cergy-Pontoise- 2 Av Adolphe Chauvin 95302, \\ Cergy-Pontoise, France \\ \{omar.moussaoui, adlen.ksentini, mohamed.naimi\} \\ adept-info.u-cergy.fr \\ ${ }^{2}$ PRiSM CNRS, Université de Versailles- 45, Av des Etats-Unis 78035, \\ Versailles, France \\ mogue@prism.uvsq. fr
}

\begin{abstract}
Multimedia applications, such as videoconferences, require an efficient management of the Quality of Service (QoS) and consist of a great number of participants which requires the use of multicast routing protocol. Unlike unicast protocol, multicast protocol handles a great number of users while minimizing both network overhead and bandwidth consumption. However, combining multicast routing and QoS guarantee is a hard challenging task, known as the delay and delay variation multicast problem. This problem is considered as an NP-complete problem, and is resolved only by heuristic solutions. In this paper, we propose a scalable multicast algorithm that tackle the delay and delay variation by exploiting the Hierarchic Tree construction concepts. In fact, the proposal algorithm guarantees QoS by: (i) reducing the network charge; (ii) decreasing the multicast delay variation. We compare the performance of our algorithm against the DDVCA (Delay and Delay Variation Constraint Algorithm) scheme and demonstrate lower multicast delay variation and efficient bandwidth utilization while maintaining lower time complexity.
\end{abstract}

\section{Introduction}

The demand for multimedia that combines audio, video and data streams over a network is quickly increasing. Among the most popular real-time interactive applications, videoconferences and games require a considerable amount of bandwidth and a great number of participants. In this context multicast is regarded as a promising solution for group multimedia applications. In fact, multicast is a bandwidth-conserving technology that reduces traffic by simultaneously delivering a single stream of information to thousands of corporate recipients or groups. Multicast delivers source traffic to multiple receivers without adding any additional burden on the source or the receivers while using the least network bandwidth of any competing technology. Multicast packets are replicated in the network through routers enabled with multicast protocol and other supporting multicast protocols resulting in the most efficient delivery of data to multiple receivers possible. All alternatives require the source to send more than one copy of the data. Some even require the source to send an individual copy to each receiver. If there are thousands of receivers, even low-bandwidth applications benefit from using multicast. High-bandwidth applications, such as H.264 video, may require a large portion of the available network bandwidth for a single stream [1]. In these 
applications, the only way to send to more than one receiver simultaneously is by using multicast. Several protocols were proposed in the literature [2], [3], [4], [5] aiming to propose a multicast protocol. However, the majority of them are not scalable, which means not adaptable to the networks of great dimension like Internet. By limiting the deployment of these protocols, the Internet is becoming the unavoidable network. Furthermore, these protocols work as best effort protocols [6], so they cannot handle sensitive traffics such as video conference and real time game. Indeed, these applications require that all the destination nodes must receive the same data simultaneously; otherwise the communication may lose the feeling of an interactive face-to-face discussion. Thus it is important to sustain good QoS support while proposing a scalable multicast protocol. This constraint is related to the multicast Delay and delay-Bounded Multicast Tree (DVBMT) problem [7]. Although this problem is considered as $N P$-complete hard problem, there are some heuristics that are proposed as a possible solution [7], [8].

In this paper we tackle the DVBMT problem by proposing a novel scalable multicast algorithm, which produces multicast tree while maintaining a good sustained QoS. The main idea is to combine a hierarchic tree construction with efficient multicast grouping concepts. Firstly, like [9], we decompose the multicast group into local groups based on delay constraint and user's station capacity. This allows us to have several groups with a reduced intra group delays. Afterwards, we select a server that minimizes the delay variation with the others selected nodes from each group. From the server's set obtained, we choose a core nodes or rendezvous points. Finally, we use both the hierarchical trees and the core nodes to connect these multicast group members. Thus, we solve the DVBMT problem by decomposing the problem into two parts: $(i)$ end to end delays which is solved by constructing local group with minimum delays; (ii) multicast delay variation solved by joining the hierarchic tree construction with the core nodes concepts.

The rest of the paper is organized as follows: In section 2, we give an overview of QoS and multicast protocols. Section 3 presents details of the proposed algorithm. Then, in section 4 , we evaluate the proposed scheme by simulation model. Section 5 concludes this paper.

\section{Multicast and QoS Overview}

Algorithms for the tree construction in multicast protocols can be categorized as follows: Source-Based Algorithms (SBA) and Core-Based Algorithms (CBA) [10].

SBA constructs a specific tree where the tree's root is the source node and the leaves are the multicast group's components. SBA is currently used as the tree construction algorithm for Distance Vector Multicast Routing Protocol (DVMRP) [2], Protocol Independent Multicast Dense Mode (PIM-DM) [3], and Multicast Open Shortest Path First (MOSPF) [4].

CBA is used in the context of many-to-many multicasts. Actually, the core-based algorithm selects a core node as multicast tree's root. Afterwards, a tree rooted at the core node is constructed to span all members in the multicast group. Therefore it is very important to select the best core node as much as possible. Thus messages generated at the source are sent to the core node, and they are distributed to destinations 
through this core node. Multicast protocols using CBA as a tree construction algorithm include Protocol Independent Multicast Sparse Mode (PIM-SM) [5] and the Core-Based Tree (CBT) protocol [11]. The core-based algorithms are highly suitable for sparse groups and scalable for large networks. They provide excellent bandwidth conservation for receivers.

In addition to the need of scalability, group based multimedia applications also demand stringent QoS requirements such as bounded end-to-end delay, multicast delay variation and the efficient use of the bandwidth. The multicast end-to-end delay stands for an upper bound of all end-to-end delays associated with the paths from the source node to each of the destination nodes. The purpose of setting this parameter is to limit the time for message transmissions in the network. If the end-to-end delay exceeds the upper bound, the message will be counted useless. The multicast delay variation is the difference of the maximum end-to-end delay and the minimum end-toend delay among the paths from the source node to all the destination nodes has to be kept within. Enabling this parameter allows all the destination nodes to receive the same data simultaneously as much as possible. The issue first defined and discussed in [7] is to minimize multicast delay variation under multicast end-to-end delay constraint. In fact, the authors tackle the DVBMT problem by proposing a heuristic solution called Delay Variation Multicast Algorithm (DVMA). DVMA constructs at first the tree by considering only the end-to-end delay constraints. Afterwards, the tree is enhanced by considering the multicast delay variation constraint. At the end, DVMA's algorithm returns a feasible tree, which minimizes the end-to-end delays and optimizes the multicast delay variation. Nevertheless, the main weakness of DVMA is time complexity. Actually, DVMA exhibits a high time complexity about $O\left(p l m n^{4}\right)$, where in the worst case, the maximum value that $p$ and $l$ can take is equal to the maximum number of paths of the tree, $m$ is the size of the multicast group $M$, and $n$ is the number of nodes in the network. Accordingly, this time complexity does not fit in modern high-speed computer network environment. Delay and Delay Variation Constraint Algorithm (DDVCA) presented in [8] aims to solve the DVBMT problem by proposing another heuristic solution with lower time complexity than DVMA. DDVCA's algorithm is based on the Core Based Tree (CBT). In fact the authors propose to build the multicast tree around one core node, which is selected as the node with the minimum delay variation with all the others nodes present in the multicast group. Thus it has been shown that DDVCA outperforms DVMA in terms of the multicast delay variation of the constructed tree. Furthermore, DDVCA shows lower time complexity than DVMA, which is equal to $O\left(m n^{2}\right)$. Nonetheless, if we consider the network utilization, we see that DDVCA exhibits high network charge around the core node. In fact, all the multicast session's packets transit through the core node, which leads to network congestion in the neighboring of the core node. Furthermore, when packets arrive to the core node from the sender, this last one resends these packets to the leaves using a unicast routing protocol. Therefore, DDVCA looses the benefits of using a multicast routing protocol.

To overcome these limitations, we propose an algorithm which allows efficient communication between the multicast group's members by supporting QoS constraints. The proposed algorithm solves the DVBMT problem by constructing a hierarchical tree based on delay and multicast delay variation. Firstly, we decompose the multicast members into a disjoint local groups based on their localization and their 
response to application QoS requirement. Thus we obtain local groups with minimum intra-group delay. Afterwards, from each group a server is selected, where the server is the node that minimizes the multicast delay variation with the others group's node. At this point we have the first level of the hierarchical tree. Secondly, we select a core node from the server sets, and this core node is the server which minimizes the multicast delay variation with the others selected server. Here we are able to construct the second level of the hierarchical tree. In fact, based on this core node we build the autonomous domain (AD). The first AD contains a set of groups, where the delay from the core node to each group's server is less than a predefined threshold. From the server sets that not belong to the first $\mathrm{AD}$, we elect another core node. This second core node is the server that minimizes the delay with the first core node. Through this second core node we create another AD. Thus we redo the procedure of AD creation until we incorporate all the local groups into different AD. At the end, we construct a hierarchical tree with core-based algorithms. However, unlike DDVCA where the tree construction is based only on one core node, we extend this construction of the tree on several core nodes. This allows us to share out the network charge around different core node, leading to minimize the bandwidth consumption. Further we limit the use of the unicast mechanism only at the intra local group communications.

\section{Description of the Proposed Algorithm}

To illustrate the proposed algorithm, we will use directed graph $G=(V, E)$ to denote a network, where $V$ is a set of nodes or routers and $E$ is a set of directed links, respectively. Each link $(i, j) \in E$ is associated with delay $d_{i j}$. The delay of a link is the sum of the perceived queuing delay, transmission delay, and propagation delay over that link. Here we note a path as sequence of nodes $u, i, j, \ldots, k, v$, where $(u, i),(i, j), \ldots,(k, v) \in E$. Let $P(u, v)=\{(u, i),(i, j), \ldots,(k, v)\}$ denotes the path from node $u$ to node $v$, so a simple path is a path where all its elements are distinct. At this point a multicast group $M \subseteq G$ is constituted by $m$ processes (participants) distributed geographically on the Internet network. Moreover, these processes participate at the same multimedia application such as videoconferencing. Note that, communication between two processes $m_{i}$ and $m_{j}$ can take different path.

\subsection{Local Groups' Construction}

Considering the high number of participants in multicast group $M$, it appears essential to divide them into local groups according to their concentration in the various areas of the Internet. This decomposition allows to: (i) efficiently use the bandwidth; (ii) reduce the consumption of resources; (iii) optimize the delay; (iiii) ensure communications between processes.

At first we begin by constructing the neighboring sets $N S_{\mathrm{i}}$ for each process $m_{\mathrm{i}}$ according to two conditions: the round-trip delay and packets Time to Live (TTL). Actually, each $m_{\mathrm{i}}$ sends a request packet along its multicast groups by using the Internet Protocol (IP) multicast addresses. In the current IP multicast architecture, a globally unique class D is allocated to each group to identify the set of destination hosts belonging to the group. Through the responses obtained from the multicast 
group, $m_{\mathrm{i}}$ selects the process $m_{\mathrm{j}}$ to include into the $N S_{\mathrm{i}}$ by checking if the $T T L_{i j}$ from $m_{\mathrm{i}}$ to $m_{\mathrm{j}}$ (decremented hop by hop) is not null and the Round-Trip Delay $R T D_{\mathrm{ij}}$ between $m_{\mathrm{i}}$ and $m_{\mathrm{j}}$ is less than a given threshold of delay SupD (1). In other word, the path from $m_{\mathrm{i}}$ to $m_{\mathrm{j}}$ is the shortest in terms of delay. According to this selection, we divide the multicast group onto a set of $N S_{\mathrm{i}}$ with low intra-communication delays.

$$
N S_{i}=\left\{m_{j} \in M / R T D_{i j} \prec S u p D \& \& T T L_{i j} \succ 0\right\}
$$

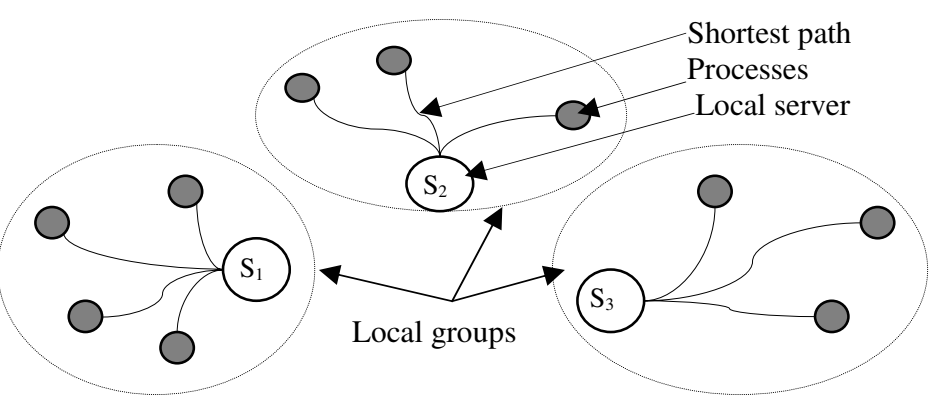

Fig. 1. Construction of local groups

At this point, each $m_{\mathrm{i}}$ has its own $N S_{\mathrm{i}}$. However, this is insufficient if we consider the different process' capacities in terms of media unit processing time and the generated rate of media units, noted $m p t_{i}$ and $\sigma_{i}$, respectively. In this context we must refine the $N S_{\mathrm{i}}$ in order to take the process's capacities into account. To this end, each $m_{\mathrm{i}}$ carries out evaluations on its capabilities of processing and buffering of media units, in order to choose the $m_{\mathrm{j}}$ which will constitute its new enhanced $N S_{\mathrm{i}}$. Here we note this enhanced $N S_{\mathrm{i}}$, by the transit group $T G_{\mathrm{i}}$. Thus each $m_{\mathrm{i}}$ elects the $m_{\mathrm{j}}$ process that composes its transit group according to both (2) and (3). In fact these two constraints allow that $m_{\mathrm{i}}$ selects the other process in respect to its capabilities to handle data flow coming from its neighbors. Thus a process $m_{\mathrm{i}}$ must be able to: $(i)$ process all media units generated in the same time by its neighbors in a time duration not exceeding the time allocated to the processing of media units; (ii) store all media units coming on different paths between $m_{\mathrm{i}}$ and its neighboring processes.

$$
\begin{aligned}
& {\left[\sum_{m_{j} \in N S_{i}} \sigma_{j}\right] * m p t_{i}<1} \\
& \sum_{m_{j} \in N S_{i}} B_{i j}\left(C_{i j}\right)<B_{i}
\end{aligned}
$$

Where $B_{\mathrm{i}}$ represents the maximum buffer space available at the process $m_{\mathrm{i}}$, and $B_{\mathrm{ij}}$ denotes the buffer size at $m_{\mathrm{i}}$ and $C_{\mathrm{ij}}$ is the path between $m_{\mathrm{i}}$ and $m_{\mathrm{j}}$. Therefore, since $m_{\mathrm{i}}$ founds that neither (2) nor (3) are feasible when adding another $m_{j}$ to the $T G_{i}$, the process stops and the $T G_{\mathrm{i}}$ is finalized. 
Once transit groups are built, each process knows the members of its own set and the paths that connect it to them. However, some processes can belong to several transit groups at the same time. To solve this problem, a mechanism must take place to remove useless connections. Each process must broadcast its transit group to its neighbors. Once these packets are received, each receiver $m_{\mathrm{i}}$ selects the maximum of the processes existing simultaneously in these groups of transit $\left(L G_{i}=\bigcap_{j \in T G_{i}} T G_{j}\right)$. If a process belongs to several local groups, then this process is placed into the smallest $L G_{\mathrm{i}}$ in size. This allows to equally balancing the process number in these groups.

Thus, the multicast group $M$ is divided into local sub-groups (Figure 1). Each element of $M$ belongs to only one local group. After that a local server and a secondary server are elected to represent each local group. In other terms, a process

Let $G=(V, E)$ a computer network and $M=\left\{m_{l}, m_{2}, \ldots, m_{m}\right\}$ a set of participants in group multicast $M$.

Begin

1. for each process $m_{i} \in M$ do //construct a neighboring set $\mathrm{NS}_{\mathrm{i}}$ of $m_{i}$

2. $N S_{i}=\left\{m_{j} \in M / R T D_{i j} \prec S u p D \& \& T T L_{i j} \succ 0\right\}$

3. end of for each $m_{i} \in M$ loop

4. for each process $m_{i} \in M$ do //construct a transit group $T G_{i}$ of $m_{i}$

5. $\sigma=0, B=0 / / \sigma$ and $B$ are temporaries variables

6. for each process $m_{j} \in N S_{i}$ do

7. $\sigma=\sigma+\sigma_{j}, B=B+B_{i j}$

8. if $\sigma^{*} m p t_{i} \prec 1$ and $B \prec B_{i}$ then $T G_{i}=T G_{i} \cup\left\{m_{j}\right\}$

9. end of for each $m_{j} \in N S_{i}$ loop

10. end of for each $m_{i} \in M$ loop

11. for each process $m_{i} \in M$ do // construction of local groups

12. $L G_{i}=\bigcap_{j \in T G_{i}} T G_{j}$

13. end of for each $m_{i} \in M$ loop

14. for each local group $L G_{i}$ do // election of local servers

15. elect local server $S_{i}$ and secondary server $S S_{i}$

16. $\Gamma=\Gamma \bigcup\left\{S_{i}\right\}$ and $k=k+1$

17. end of for each local group $L G_{i}$ loop

18. for each local server $S_{i} \in \Gamma$ do

19. for each $m_{j} \in L G_{i}$ do

20. $S_{i}$ joins $m_{j}$ by the path which has a minimum round-trip delay.

21. end of for each $m_{j} \in L G_{i}$ Loop

22. end of for each local server $S_{i} \in \Gamma$

Fig. 2. Algorithm of local groups' construction 
communicates with the other participants of the multicast group only through the server of its local group. The local and secondary servers are the processes that minimize the multicast delays with the others process, meanwhile these servers must have the maximum processing capacity. Note that, the principal role of the secondary server is to replace the local server if this last leaves the multicast group or it crashes (break down). For completeness, we draw in Figure 2 the local group algorithm construction.

\subsection{Multicast Tree Construction Between Servers}

Let us consider $k$ local groups are built and each group has its local server (Figure 1). Here $\Gamma=\left\{S_{1}, S_{2}, \ldots, S_{k}\right\}$ is the set of these local servers distributed in different networks. It is important to note that the number of these servers can be very high and several sources can belong to the same multicast group. Accordingly, it is necessary to build a multicast tree which links these servers while reducing the multicast group's participants. In this context, like DDVCA, we propose the use of core-based tree. However, we based the tree construction on several core nodes instead of one, aiming to avoid congestion problem. These core nodes are selected from $\Gamma$, by considering the servers which minimize the multicast delay variation with the others $\Gamma$ 's servers.

Table 1. Packets and data structures employed

\begin{tabular}{|l|l|l|}
\hline Type & Arguments & function \\
\hline INIT & $\begin{array}{l}\text { adr } S_{i}: \text { address of the local server } S_{i} \\
\text { TTL: } \text { Time to Live } \\
a d r M: \text { address of the group multicast }\end{array}$ & $\begin{array}{l}\text { initialization } \\
\text { packet }\end{array}$ \\
\hline ACK & $\begin{array}{l}a d r S_{j}: \text { address of the sender } S_{j} \\
a d r S_{i}: \text { address of the receiver } S_{i}\end{array}$ & $\begin{array}{l}\text { acknowledgement } \\
\text { packet }\end{array}$ \\
\hline SUCC & $\begin{array}{l}a d r S_{i}: \text { address of the new indicated core node } S_{\mathrm{i}} \\
\text { \$d } N_{j}: \text { address of the core node predecessor of } S_{\mathrm{i}} \\
\text { mous domains of core nodes already created } \\
\text { MAT: matrix allowing to store the minimum delay between } \\
\text { the elements of } \$ \text { and core nodes in the multicast tree }\end{array}$ & successor packet \\
\hline
\end{tabular}

Initially, each local server broadcasts hop by hop an initialization packet INIT (Table1) at the multicast group $M$ address. All members of $M$ that are not servers delete the received packet, while the other nodes (server $S_{\mathrm{i}}$ ) carry out the following operation:

- Response to the sender server $S_{\mathrm{j}}$ with an acknowledge packet $A C K$.

- Compute the minimum delay $d_{\min }\left(S_{i}, S_{j}\right)$ between it and $S_{\mathrm{j}}$ by using Dijkstra's algorithm [12].

- Memorize the address of $S_{\mathrm{j}}$ and the value of delay $d_{\min }\left(S_{i}, S_{j}\right)$.

Finally, each server $S_{\mathrm{i}}$ calculates its multicast delay variation through:

$$
\delta_{S_{i}}=\max \left\{\left|d_{\min }\left(S_{i}, S_{j}\right)-d_{\text {min }}\left(S_{i}, S_{k}\right)\right| / \forall S_{j}, S_{k} \in \Gamma, j \neq k\right\}
$$


From this point each server exchanges the multicast delays variations values with the other local servers. Thus the server that minimizes the multicast delay variation will be elected as the first core node and noted $C N_{1}\left(\delta_{C N_{1}}=\min \left\{\delta_{S_{i}} / S_{i} \in \Gamma\right\}\right)$.

Afterwards, the first core node $C N_{1}$ builds its Autonomous Domain $A D_{1}$ by selecting all local servers of $\Gamma$ that are accessible through a delay time lower than a given threshold of delay $D$ (the threshold $D$ is selected by taking account of the network extent). These allow us to build an Autonomous Domain with a minimum intra-delay communications.

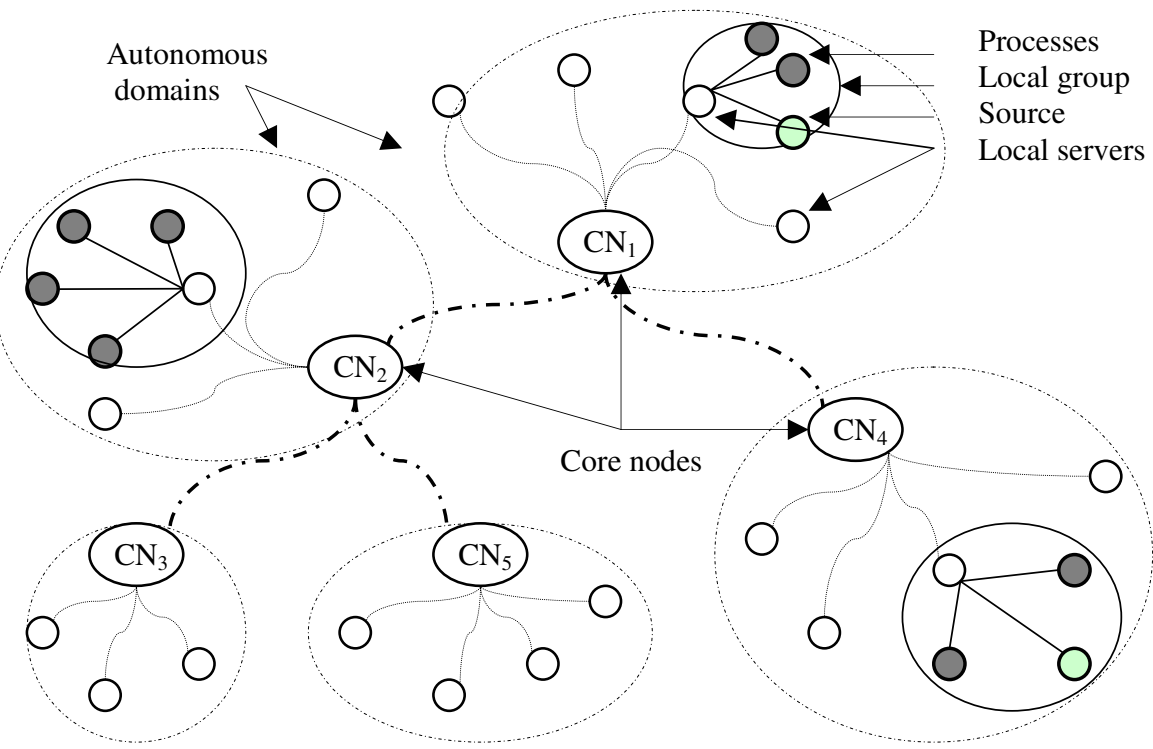

Fig. 3. Hierarchical structure of multicast group

Table 2. Delays between core nodes and servers of $\$$

\begin{tabular}{|c|c|c|c|}
\hline & $S_{\mathrm{i}}$ & $S_{\mathrm{j}}$ & $\ldots$ \\
\hline$C N_{1}$ & $d_{\min }\left(C N_{1}, S_{i}\right)$ & $d_{\min }\left(C N_{1}, S_{j}\right)$ & \\
\hline$C N_{2}$ & $d_{\min }\left(C N_{2}, S_{i}\right)$ & $d_{\min }\left(C N_{k}, S_{i}\right)$ & \\
\hline$\ldots$ & & & \\
\hline
\end{tabular}

Here, all the other servers that are not in $C N_{1}$ 's domain will be stored in $\$$ such as $\mathrm{AD}_{1}: \$=\Gamma \backslash A D_{1}$. Then, $C N_{1}$ stores the minimum delay between it and all servers of $\$$ in the matrix MAT (Table 2). After that $C N_{1}$ 's $\mathrm{AD}$ is built, and $C N_{1}$ gives the relay to another server in order to build the next AD. This is made by sending a SUCC packet to the nearest server $S_{\mathrm{i}}$ belonging to $\$$. In other words, $S_{\mathrm{i}}$ is the server which minimizes the delay with $C N_{1}\left(d_{\min }\left(C N_{1}, S_{i}\right)\right)$. Finally, $C N_{1}$ joins the members of its autonomous domain $A D_{1}$ via the shortest path tree while the root is $C N_{1}$. 
Meanwhile, when a local server $S_{\mathrm{i}}$ receives a $S U C C$ packet from $C N_{1}$, this means that $S_{\mathrm{i}}$ is promoted as new core node noted $C N_{2}$. Accordingly $S_{\mathrm{i}}$ carries out the following operations:

- Select all servers of $\$$ (the set of servers which are not in the domain of $C N_{1}$ ) having a delay smaller or equal to the threshold $D$ and put them into its autonomous domain $A D_{2}: A D_{2}=\left\{S_{i} \in \$ / d_{\text {min }}\left(C N_{2}, S_{i}\right) \prec D\right\}$.

- Remove from $\$$ the selected servers: $\$=\$ \backslash D_{2}$.

- Remove the columns of the matrix MAT corresponding to the elements of its domain.

- Add a line into MAT to store the minimum delay between it and all servers of \$: which do not belong to any domain.

- Search the smallest value of delays in MAT and take the pair $\left(C N_{\mathrm{p}}, S_{\mathrm{q}}\right)$ corresponding to this value. In other terms, $C_{2}$ selects the server $S q$ which minimizes the delay with already created core nodes $\left(C N_{l}\right.$ or $\left.C N_{2}\right)$.

- Send a $S U C C$ packet to the new core node $S_{\mathrm{q}}$ noted $\mathrm{CN}_{3}$.

Finally, $C N_{2}$ joins its domain members and the predecessor core node $C N_{1}$ via the shortest path tree where the root is $C N_{2}$. Once the new core node $C_{3}$ receives the

Let $\Gamma=\left\{S_{1}, S_{2}, \ldots, S_{k}\right\}$ be a set of elected local servers.

Begin

1. for each local server $S_{i} \in \Gamma$ do

2. $d_{\min }\left(S_{i}, S_{j}\right)=$ the minimum delay between $S_{i}$ and $S_{j}$, where $S_{j} \in \Gamma$ (the minimum delay is computed by Dijikstra' Algorithm)

3. end of for each local server $S_{i} \in \Gamma$ loop

4. for each local server $S_{i} \in \Gamma$ do //calculate the multicast delay variation of $S_{i}$

5. $\delta_{S_{i}}=\max \left\{\left|d_{\min }\left(S_{i}, S_{j}\right)-d_{\min }\left(S_{i}, S_{k}\right)\right| / \forall S_{j}, S_{k} \in \Gamma, j \neq k\right\}$

6. end of for each local server $S_{i} \in \Gamma$ loop

7. NewCN $\leftarrow S_{i}$, where $\delta_{S_{i}}=\min \left\{\delta_{S_{j}} / S_{j} \in \Gamma\right\} \quad / /$ the server which has a minimal multicast delay variation represents the first core node

8. $\$ \leftarrow \Gamma$, Predecessor $\leftarrow$ New $C N$ and $\operatorname{Set} C N \leftarrow \varnothing$

9. while $\$ \neq \varnothing$ do

10. $C N_{i} \leftarrow$ NewCN, SetCN $\leftarrow$ SetCN U $\left\{\mathrm{CN}_{\mathrm{i}}\right\}$, Pred $\left(C N_{i}\right) \leftarrow$ Predecessor

11. $A D_{i}=\left\{S_{j} \in \Gamma / d_{\text {min }}\left(C N_{i}, S_{j}\right) \prec D\right\} / / C N_{i}$ builds its autonomous domain

12. $C N_{i}$ joins member of its domain $A D_{i}$ and its predecessor $\operatorname{Pred}\left(C N_{i}\right)$ by the shortest path tree which root is $C N_{i}$

13. $\$ \leftarrow \$ \backslash A D_{\mathrm{i}}$, removes $A D_{i}$ from columns of $M A T$ and adds line corresponding to $\left\{d_{\text {min }}\left(C N_{i}, S_{i}\right) / S_{i} \in \$\right\}$ in $M A T$

14. NewCN $\leftarrow S_{q}$ and Predecessor $\leftarrow C N_{p}$, where $d_{\min }\left(C N_{p}, S_{q}\right)=\min \left\{\operatorname{MAT}\left(C N_{j}, S_{k}\right) / S_{k} \in \$, C N_{j} \in \operatorname{Set} C N\right\}$

15. end of while loop

End of the algorithm

Fig. 4. Algorithm for multicast tree construction between local servers 
packet $S U C C$, it makes the same thing as $C_{2}$. Note that the AD building process is ended when $\$$ is empty or in other words, until all servers are connected to the multicast tree (Figure 3). For completeness we draw in Figure 4 the multicast tree construction algorithm.

\subsection{The Time Complexity of the Algorithm}

In order to determine the complexity of the proposed algorithm, we consider the following lemma:

Lemma. The worst case complexity of the algorithm is $O\left[(k+1) n^{2}\right]$, where $k$ is the number of selected local servers and $n$ is the number of nodes in the network.

Proof. The proposed algorithm time complexity is the sum of the time complexity of building the local groups (Figure 2) and the time complexity of building the multicast tree between the servers (Figure 4). On the one hand, the time complexity of building the local groups is in the worst case $O\left(\mathrm{~m}^{2}\right)$, where $m$ is the participants' number in the group multicast $M$. In fact, the time complexity of constructing a neighbouring set $N S_{i}$ of process $m_{i}$ (Figure 2, line 2) is $O(m)$. Given that our algorithm executes the loop from line 1 to line 3 once for each process $m_{\mathrm{i}}$ belonging to $M$. $\left(m_{i} \in M\right)$, the time complexity of lines 1-3, therefore, is $O\left(\mathrm{~m}^{2}\right)$. Here, the transit groups are constructed from line 4 to line 10, so during one iteration of the outer loop (4-10), the lines 7 and 8 are executed at most $m$ times $\left(\forall m_{i} \in M,\left|N S_{i}\right| \prec m\right.$ ). Accordingly, the time complexity of lines 4-10 is $O\left(\mathrm{~m}^{2}\right)$. Further the loop from line 11 to line 13 constructs local groups. Since the time $O(m)$ is required at most in line $12\left(\left|T G_{i}\right|<<m\right)$, then the time complexity of lines 11-13 is $O\left(\mathrm{~m}^{2}\right)$. From this point the number of iterations required to elect local and secondary servers for each local group $L G_{i}$ is $\left|L G_{i}\right|$. If one consider that $m=\left|L G_{l}\right|+\left|L G_{2}\right|+\ldots+\left|L G_{k}\right|$, then the time complexity of lines 14-17 is $O(m)$. Finally, the time complexity of loop from line 18 to line 22 is $O(\mathrm{~km})$. Since $m$ is much higher than $k(m>>k)$, then the time complexity of constructing local groups is $3^{*} O\left(\mathrm{~m}^{2}\right)+O(\mathrm{~km})+O(\mathrm{~m})=O\left(\mathrm{~m}^{2}\right)$.

On the other hand, it is easily observed that the execution time of the multicast tree construction algorithm (Figure 4) is mainly spent on the loop between lines 1 and 3, namely on calculating the minimum delay between local servers. The time complexity of computing these minimum delays by Dijkstra's Algorithm is $O\left(n^{2}\right)$ [12]. Given that the proposed algorithm executes the loop from line 1 to line 3 once for each local server, the time complexity of lines $1-3$, therefore, is $O\left(k n^{2}\right)$, where $k$ is the number of elected local servers. Line 4 through line 6 compute the multicast delay variation for each local server $S_{i} \in \Gamma$, so if we consider that the time $O(k)$ is required in line 5, then the time complexity of lines 4-6 is $O\left(k^{2}\right)$. The design of the local server which has a minimal multicast delay variation as a first core node is in line 7 . Thus, the time complexity required for execute line 7 is $O(k)$. Here, the loop from line 9 to line 15 connects all the local servers to the multicast tree. Line 11 requires $k$ iteration to construct an autonomous domain $A D_{i}$ for each selected core node $C N_{i}$. In line 12 the selected core node $C N_{i}$ joins members of its domain $A D_{i}$ and its core node predecessor $\operatorname{Pred}\left(C N_{i}\right)$, within a number of iterations less than $k\left(\left|A D_{i}\right|+1<k\right)$. Further line 14 selects the new core node from the set of servers which are not belonging to any cre- 
ated autonomous domain through $k^{2}$ times. Thereby, since the loop between lines 9 and 15 is executed at most $k$ times, then overall time complexity of lines $9-15$ is $O\left(\mathrm{k}^{3}\right)$. Finally, the time complexity of constructing multicast tree between servers is $O\left(k n^{2}\right)+O\left(k^{2}\right)+O(k)+O\left(k^{3}\right)=O\left(k n^{2}\right)$, because $n$ is much higher than $k(n>>k)$.

At this point by considering that $O\left(m^{2}\right)+O\left(k n^{2}\right)=O\left[(k+1) n^{2}\right]$, because the number of destinations nodes $m$ is lower than the number of nodes in the network $n$, the overall time complexity of the proposed algorithm is $O\left[(k+1) n^{2}\right]$. Since $k<<m$, then our algorithm shows lower time complexity than DDVCA, which is equal to $O\left(m n^{2}\right)$.

\section{Simulations and Analysis}

In order to evaluate the advantages of the proposed scheme, we have constructed a set of simulation using ns-2 (Network Simulator) [13]. We compare the proposed algorithm with DDVCA. The simulations focus on the protocols' abilities to maintain low multicast delay variation while minimizing the bandwidth consumption. During the simulation, we deliberately change the network topology by changing the network's size (500 and 1000 stations) in order to evaluate the ability of the proposed scheme to suit different network configuration. Further, the destination nodes in the multicast group represent $50 \%$ of the network size, while 10 source nodes are chosen randomly. Each run consists of 100 second, where each source generates 20 (media units/s). Note that the media unit size is 500 bytes. For completeness the scheduling of multimedia flows' diffusion is shown in Table 3 .

Table 3. Scheduling of diffusion multimedia stream

\begin{tabular}{|c|c|c|c|c|c|c|c|c|c|c|}
\hline Sources & 1 & 2 & 3 & 4 & 5 & 6 & 7 & 8 & 9 & 10 \\
\hline Begin (s) & 0 & 10 & 20 & 30 & 40 & 50 & 60 & 70 & 80 & 90 \\
\hline End (s) & 100 & 100 & 100 & 100 & 100 & 100 & 100 & 100 & 100 & 100 \\
\hline
\end{tabular}

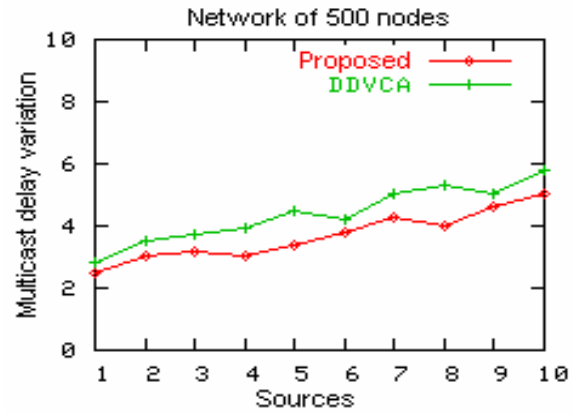

Fig. 5. Multicast delay variation's average 500 stations

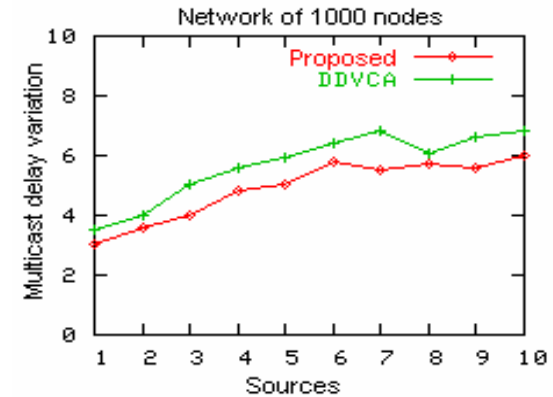

Fig. 6. Multicast delay variation's average 1000 stations

Figures 5 and 6 represent the multicast delay variation average when using 500 and 1000 stations, respectively. It's clearly seen that our mechanism outperforms the DDVCA mechanism in both situations. This is expected as our algorithm constructs both the local group and the autonomous domain according to the multicast delay 
variation constraint. In contrast DDVCA takes this constraint into account only through the choice of the core node. Furthermore, it is important to note that our scheme's gain in multicast delay variation over DDVCA is roughly $1 \mathrm{sec}$, which is very high if we consider the case where the flows represent a video-based session. In fact, this translates into a high jitter entailing devastating consequences on the perceived video quality at the receiver.

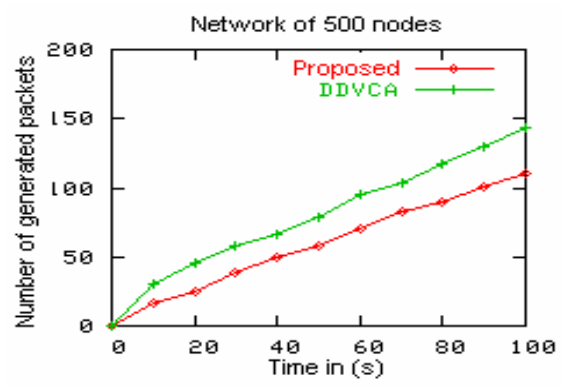

Fig. 7. Bandwidth consumption 500 stations

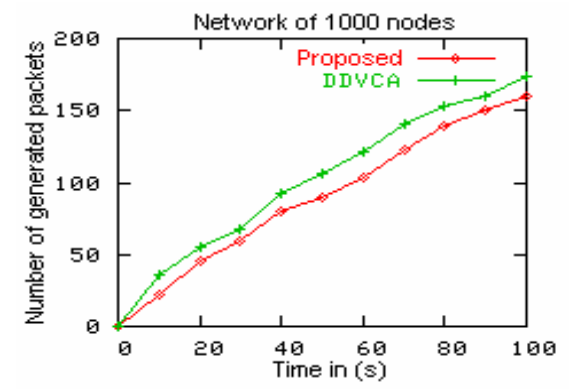

Fig. 8. Bandwidth consumption 1000 stations

Figures 7 and 8 show the bandwidth consumption of both the proposed scheme and DDVCA. Note that these graphs are obtained by computing periodically $(10 \mathrm{~s})$ the packets' number generated by both the proposed scheme and DDVCA. Actually, DDVCA uses more bandwidth than the proposed scheme. This is caused by the fact that DDVCA avoids using the unicast packets only between the senders and the core node. In contrary, the proposed scheme uses the unicast packets only in the intra-local group communication. Indeed, the unicast packets increase the bandwidth consumption.

Additionally, to see the influence of the multicast group's size, we have increased the number of nodes in the network (1000 stations). As indicated, it is easy to notice that the proposed algorithm is always better than the DDVCA.

\section{Conclusion}

In this paper we have proposed a novel multicast tree construction in order to solve the so called delay and multicast delay variation problem. The proposed scheme offers an improved ability to minimize the multicast delay variation as well as the bandwidth consumption while having a lower time complexity.

Simulations have shown that the proposed mechanism achieves numerous performance gains over the DDVCA. In addition to minimize the multicast delay variation, the proposed scheme improves efficiently the bandwidth utilization by minimizing the packets' number in the network. Furthermore, the proposed algorithm exhibits a time complexity lower than DDVCA, $O\left(m n^{2}\right)$ and $O\left[(k+1) n^{2}\right]$ respectively $(k<<m)$. Our future works will focus on the implementation of our algorithm in real network configuration. Furthermore, we will also extend our proposal with a QoS management-based such as Diffserv. 


\section{References}

1. A. Ksentini et al. "Novel Architecture for reliable H.26L video transmission over IEEE 802.11e", Proc. IEEE PIMRC'05, Barcelona, Spain.

2. D. Weitzman, C. Partridge, "Distance Vector Multicast Routing Protocol", RFC 1075, November 1998.

3. S. Deering et al., "Protocol Independent Multicast-Dense Mode (PIM-DM): Protocol Specification", RFC 2365, July 1998.

4. J. Moy, "Multicast Extension to OSPF", RFC 1584, Mars 1994.

5. D. Estrin et al., "Protocol Independent Multicast-Sparse Mode (PIM-SM): Protocol Specification", IETF RFC 2362, June 1998.

6. A. Striegel and G. Manimaran, "Survey of QoS Multicasting Issues", IEEE Communications Magazine, June 2002, pp. 82-87.

7. G. N. Rouskas, I. Baldine, "Multicast routing with end-to-end delay and delay variation constraints", IEEE JSAC, April 1997, pp. 346-356.

8. P.-R Sheu, S.-T. Chen, "A fast and efficient heuristic algorithm for the delay and delay variation bound multicast tree problem", Information Networking, Proc. ICOIN-15, January 2001, pp. 611-618.

9. A. Benslimane, O. Moussaoui, "A scalable Multicast Protocol with QoS guarantees", Proc. of IEEE/IFIP Net-Con'2003. Muscat, Oman. October 2003.

10. B. Wang and J. C. Hou, "Multicast Routing and its QoS Extension: Problems, Algorithms, and Protocols", IEEE Networks, January/ February 2000.

11. A. Ballardie, "Core Based Trees (CBT Version 2) Multicast Routing: protocol specification," IETF RFC 2189, September 1997.

12. E. W. Dijkstra, A note on two problems in connection with graphs, Numeric Mathematic, vol. 1, 1959, pp. 269-271.

13. Network Simulator 2, ns-2, http://www.isi.edu/nsnam. 